\title{
Priming the holiday spirit: Persistent activation due to extraexperimental experiences
}

\author{
JenNifer H. CoANE \\ Colby College, Waterville, Maine \\ AND \\ DAVId A. BALOTA \\ Washington University, Saint Louis, Missouri
}

\begin{abstract}
The concept of activation is a critical component of many models of cognition. A key characteristic of activation is that recent experience with a concept or stimulus increases the accessibility of the corresponding representation. The extent to which increases in accessibility occur as a result of experiences outside of laboratory settings has not been extensively explored. In the present study, we presented lexical stimuli associated with different holidays and festivities over the course of a year in a lexical decision task. When stimulus meaning and time of testing were congruent (e.g., leprechaun in March), response times were faster and accuracy greater than when meaning and time of test were incongruent (e.g., leprechaun in November). Congruency also benefited performance on a surprise free recall task of the items presented earlier in the lexical decision task. The discussion focuses on potential theoretical accounts of this heightened accessibility of time-of-the-year-relevant concepts.
\end{abstract}

Activation is a fundamental construct in many classic models of cognition (e.g., Anderson, 1983; Collins \& Loftus, 1975; Dell, 1986). In its simplest instantiation, a concept or mental representation is activated directly, through the presentation of an eliciting stimulus, or indirectly, through the presentation of a similar or related stimulus. An activated representation results in shorter response times (RTs) and greater accuracy relative to a representation that is not activated above its baseline level. For example, in semantic and repetition priming experiments, a target (e.g., DOG) preceded either by itself $(d o g)$ or a related prime (cat) produces facilitation relative to when it is preceded by an unrelated prime (chair). Activation, as it is conceptualized by most models, is assumed to be short-lived, such that representations return quickly to baseline, through rapid decay or because of a more active dampening process (e.g., Anderson, 1983; Dell, 1986). Some degree of attention to the stimulus is required to keep it activated in order to avoid the overload of countless sources of activation, at least in rich linguistic contexts (e.g., Balota \& Lorch, 1986; Bowers, 2000). However, there is also evidence that conscious processing of stimuli is not necessary for representations to become active (e.g., Bodner \& Masson, 1997), although, for masked stimuli, the activation is often very short-lived.

An alternative account proposes that, instead of activation of abstract mental representations, priming effects are the result of retrieval processes (Tenpenny, 1995; Whittlesea \& Jacoby, 1990). Recent experience with a stimulus produces a memory trace, which is retrieved to facilitate processing on a subsequent encounter with the same or a similar stimulus (see Hintzman, 1986). Over time, the traces decay. A key difference between the activation and retrieval accounts is in the nature of the representations: Whereas activation accounts assume the existence of stored, relatively abstract representations that are susceptible to changes in accessibility over time, retrieval accounts do not necessarily posit the existence of abstract representations but assume that episodically laid memory traces become more accessible following recent or frequent experience (see Neely, 1991, for a discussion of these theoretical perspectives).

Given the amount of stimuli that an individual is exposed to on a daily basis, it seems plausible that not every stimulus would undergo a measurable change in activation or accessibility, but only those stimuli that are relevant to current processing would yield measurable changes in activation that extend across situational contexts. To our knowledge, the question of how naturalistic experience with a stimulus (i.e., exposure outside of an experimental setting) influences accessibility has received relatively little empirical attention in the literature. Furthermore, the extent to which a concept's accessibility changes over time and persists across different contextually defined experiences has been somewhat unexplored. The experiments most relevant to this issue are those in which stimuli are repeated in different experimental contexts. In this light, it is interesting to note that Oliphant (1983) found no repetition priming from words embedded in experimental instructions in a subsequent lexical decision task (LDT), 
suggesting limited activation. Thus, one might expect that the dampening parameters or decay mechanisms are sensitive to changes in context and that, once a concept is no longer relevant, its activation level quickly reverts to baseline. Similarly, a retrieval account would posit that the extent to which a trace is laid down would depend on the degree to which it was originally encoded. Because context is stored as part of the memory trace, changes in context should decrease the magnitude of priming effects due to context-dependent retrieval mechanisms. Indeed, there is a rich history that retrieval processes are sensitive to contextual changes, as is evidenced by the literature on encoding specificity (e.g., Tulving \& Thomson, 1973) and transfer appropriate processing (e.g., Morris, Bransford, \& Franks, 1977).

The question in the present study is simple: To what extent are there lingering changes in the accessibility of concepts due to recent extraexperimental experience? For example, do shamrock, fireworks, and turkey have differential states of accessibility in March, July, and November, respectively? Hence, we examined whether seasonspecific words change their accessibility as a function of the time of the year, as would be reflected by simple lexical decision performance. From a theoretical perspective, the question is whether exposure to season-specific themes actually alters the accessibility of season-relevant information. Given the richness of experiences to which individuals are exposed in naturalistic contexts and the risk of overloading the system, one might expect little persistent accessibility/activation outside of a local context. However, if activation or retrieval of memory traces are to be considered viable accounts of priming and not limited to specific experimental paradigms, it seems critical to demonstrate that such processes do occur outside of the laboratory. Indeed, it is at least possible that low-level activation may persist from highly interconnected seasonrelated networks, or schemata, and may modulate the accessibility of related concepts.

Because of the possibility that not all of the participants celebrated a particular holiday, we also included a questionnaire to assess individual differences. One might expect that the degree to which an event is central or salient to an individual would modulate the magnitude of priming effects. In fact, there is evidence, for example, that individuals with alcohol dependency or eating disorders respond differently to alcohol or food-related stimuli than control individuals (Green \& Rogers, 1993; Hill \& Paynter, 1992).
We also administered an unexpected free recall test after the LDT to assess the role of intentional retrieval processes in modulating the sensitivity to such activation (Meade, Watson, Balota, \& Roediger, 2007). Meade et al., using the Deese/Roediger-McDermott (DRM) paradigm for eliciting false memories (Roediger \& McDermott, 1995), found evidence of persistent activation of nonstudied foils semantically related to studied targets only when participants were given intentional retrieval instructions. These results suggest that rather than persistent activation from the time of study or from the original processing episode, reactivation of a memory trace due to intentional retrieval plays a critical role in eliciting false memories in the DRM paradigm (but see Tse \& Neely, 2005). Thus, one may not observe season priming in lexical decision when there is no utility in reactivating season-relevant concepts in an LDT, but there may be an influence when attention is directed to such information during a recall test. Alternatively, if there is persistent activation of concepts or if more traces are laid down as a result of naturalistic experience, one should find facilitation even in an LDT for season-relevant words.

\section{METHOD}

\section{Participants}

The participants were 204 undergraduate students recruited through the psychology research participation pool at Washington University in Saint Louis. Data collection took place throughout the year, and testing was scheduled to coincide with major holidays, festivities, or academically salient events such as finals week. Between 12 and 58 participants were tested at each point in time, and none participated at multiple time points. Eight participants' data were omitted from the analyses because of high error rates (greater than $25 \%$ ) or unusually long RTs relative to the rest of the sample (i.e., RTs that exceeded the group average by $2.5 \mathrm{SDs}$ or more). The participants received course credit or $\$ 5$.

\section{Materials}

There were 98 critical targets: 28 words related to academic events (e.g., exam, study) and 70 words related to various holidays and festivities (e.g., bunny for Easter, pilgrims for Thanksgiving, haunted for Halloween). See Table 1 for sample stimuli.

Stimuli were considered congruent when the meaning of the stimulus and the time of testing matched (e.g., leprechaun was congruent when testing occurred in mid-March) and incongruent when there was a mismatch between stimulus meaning and time of testing (e.g., leprechaun was incongruent at all testing points other than midMarch). On average, the congruent periods lasted between 2 and 3 weeks, although there was some variability due to factors such as upcoming holidays, the end of a semester, and so on. All of the

Table 1

Sample Stimuli Used in the Lexical Decision Task

\begin{tabular}{lll}
\hline Stimulus Category & \multicolumn{1}{c}{ Sample Stimuli } & \multicolumn{1}{c}{ Congruent Time of Testing } \\
\hline Independence Day & fireworks, July, independence & June 25-July 7 \\
Christmas/Hanukkah & eggnog, nutcracker, reindeer & December 3-December 15 \\
Easter & bunny, lamb, Lent & March 5-March 26 \\
Halloween & ghost, haunted, October & October 22-November 2 \\
Mardi Gras & beads, carnival, parade & February 1-February 19 \\
St. Patrick's Day & Irish, leprechaun, shamrock & March 5-March 26 \\
Thanksgiving & November, pilgrim, turkey & November 12-November 30 \\
Valentine's Day & cupid, flowers, valentine & February 1-February 19 \\
Academic & assignment, deadline, textbook & December 3-December 15 and April 16-April 30 \\
\hline
\end{tabular}


participants responded to all of the stimuli. Thus, season-congruent items served as their own baseline in the incongruent times of the year. Ninety-eight pseudohomophonic (e.g., brane) length-matched nonwords were included.

A posttest questionnaire was developed to assess the degree to which the participants were concerned about academic performance (questions included how often they studied, what their grade point average was, etc.), which holidays they celebrated, and how important these holidays were to them.

\section{Procedure}

The participants were tested individually or in small groups. A 10 -item practice list and 16 buffer items matched in length and frequency to the critical targets preceded the critical trials. The stimuli were presented one at a time in a newly randomized order for each participant. Each item remained visible until a response was made, after which a 1,000-msec intertrial interval began. After the LDT, the participants were administered the questionnaire and an unexpected final free recall test of the items from the LDT. The experiment lasted approximately $30 \mathrm{~min}$.

\section{RESULTS}

\section{LDT}

Mean correct response latencies were based on RTs between 250 and 2,000 msec and within $2.5 \mathrm{SDs}$ of each participant's mean (3.9\% of trials were omitted). To examine potential within-list priming (i.e., shorter RTs to stimuli in the second half of the list due to priming from items earlier in the list), list half (first vs. second) was included as a factor.

RT and error data were submitted to congruency $\times$ list half ANOVAs. All effects were significant at the .05 level unless otherwise specified. In RTs, the effect of list half was not significant, nor did it interact with congruency, $(F<1)$. As is shown in Figure 1, congruency clearly influenced response latencies $[F(1,195)=10.5, d=.09]$. Because different participants contributed at different points of the year, we also $z$ scored the RTs to take into consideration individual differences in overall response latency and standard deviations (see Faust, Balota, Spieler, $\&$ Ferraro, 1999). The results from this analysis yielded the same pattern $[F(1,195)=11.6, d=.24] .{ }^{1}$

As a stronger test of within-list priming, we compared responses to the very first congruent and incongruent tri- als for each participant. Of course, because we were examining a single response per participant per condition, the power in this analysis was quite low (the observed power was .37). Congruent items $(M=665 \mathrm{msec})$ were still responded to faster than incongruent items $(M=700 \mathrm{msec})$. The difference approached significance $[t(195)=1.64$, $p=.05$ (one-tailed), $d=.08]$, suggesting that the congruency effect was indeed due to extraexperimental priming and not to within-list priming.

Fewer errors were made to congruent $(M=.056$, $S E=.004)$ than to incongruent $(M=.067, S E=.003)$ $[F(1,195)=5.8, d=.21]$ targets. Again, there was no evidence of an interaction with list half $(F<1)$.

\section{Free Recall}

Because of experimenter error, recall data were available from 176 participants. Congruent targets $(M=.18$, $S E=.01)$ were recalled better than incongruent targets $(M=.13, S E=.006)[t(175)=5.4, d=.44]$. We examined intrusion errors to determine whether these effects were due just to a type of season-related response bias. Intrusions were coded by the experimenters as related (e.g., tree was a Christmas intrusion) or unrelated using a loose criterion, such that even fairly remote relations were considered related, and as congruent or incongruent. Slightly more incongruent related intrusions were made $(M=.21$ per participant) than congruent related intrusions $(M=$ .08) $[t(175)=2.2]$. Thus, the participants did not intrude words that were more relevant at a given point of year, suggesting that the congruency effect reflects changes in accessibility, rather than changes in response bias.

We tested the possibility that the congruency effect was primarily driven by a subset of items - specifically, the lowest frequency targets (e.g., leprechaun, shamrock, eggnog). Low-frequency words typically yield larger priming effects than high-frequency words (e.g., Balota \& Spieler, 1999). However, a regression analysis on the congruency effect in the LDT using length, orthographic $N$ (the number of words created by changing a single letter in a target; Coltheart, Davelaar, Jonasson, \& Besner, 1977), and HAL log frequency estimates obtained from the English Lexicon Project (ELP; Balota et al., 2007) suggested that

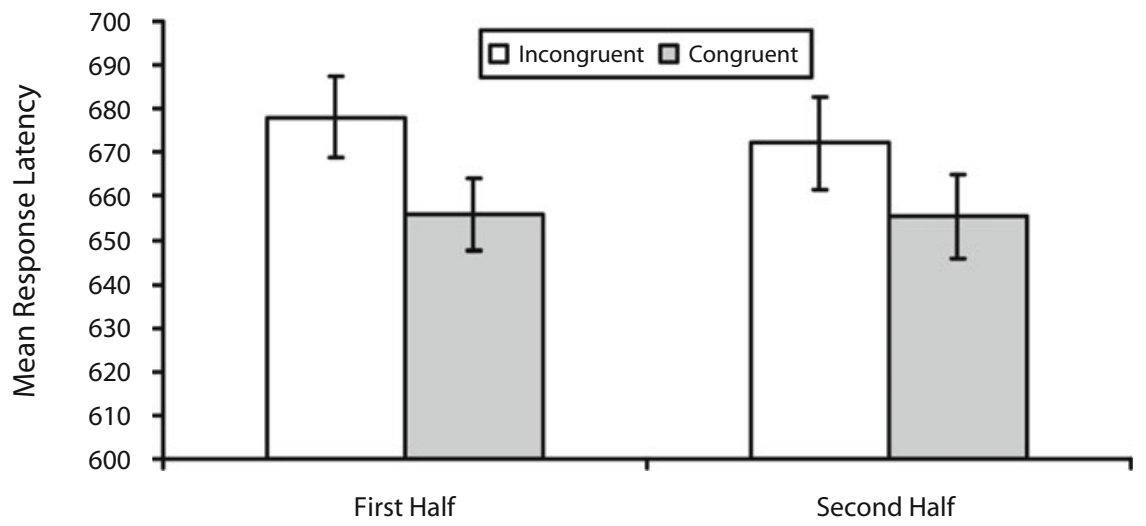

Figure 1. Mean response latencies (in milliseconds) as a function of congruency and list half. Error bars represent the standard errors of the means. 
word frequency did not predict the congruency effect in RTs, $z$-transformed RTs, or errors in LDT performance (all $p \mathrm{~s}>.15$ ). A similar analysis on the recall data indicated that there was no influence of frequency. The lack of a frequency effect was not likely due to a restriction of range, since HAL log frequency ranged from 3.09 to 12.12 , and these items produce large frequency effects in lexical decision performance in the ELP (see Balota, Cortese, Sergent-Marshall, Spieler, \& Yap, 2004). No other analyses at the item level yielded significant effects.

\section{Individual Differences}

Responses to the questionnaire were used to obtain a composite score of academic achievement. The correlation between composite score and recall of the finalsrelevant targets was reliable $[r(176)=.18, p<.05]$, and this relationship held after controlling for overall recall, indicating that the individuals with higher achievement recalled more academically relevant words. The composite score did not correlate with LDT performance. We next analyzed responses in the LDT as a function of whether the participants listed a given holiday as a favorite. In general, there was a trend for the participants to respond faster to words related to their favorite holidays (regardless of congruency). Although not significant in any of the comparisons, the facilitation ranged from 7 to $33 \mathrm{msec}$ in five of the eight festivities tested. Turning to recall performance, a significant benefit did emerge for the Easter targets $[t(174)=2.7]$. The differences for Valentine's Day and St. Patrick's Day targets approached significance in recall performance ( $p=.11$ and $p=.10$, respectively). Thus, although individual differences might have contributed to the congruency effect, it appears that it was not driven entirely by the individuals who focused attention on specific holidays or events.

\section{DISCUSSION}

The present results are quite clear in indicating persistent influences of seasonal events on the accessibility of concepts that are more or less salient (depending on the time of year). This pattern was found in both a speeded LDT and in memory performance. Congruent targets were recognized faster and more accurately than incongruent targets, and congruent targets were recalled more than incongruent targets. In LDT, the effect was present as early as the first trial, suggesting that, indeed, the congruency effect was due to extraexperimental factors and not to within-list priming. To our knowledge, this is the first report of persistent changes in accessibility levels of representations due to extraexperimental, time-of-the-year experiences.

These results suggest that the "holiday spirit" might reflect persistent, low-level activation and/or changes in accessibility of concepts in the cognitive system, consistent with the predictions of general models of cognition that incorporate an activation mechanism as a critical component. As was noted earlier, one concern is that activation effects are usually short-lived, on the order of a few minutes or less, so the rather persistent conceptual priming observed here appears to be inconsistent with a simple activation account. However, we believe that the effects observed here reflect the influence of an interconnected thematic network or schema reflecting well-established holiday-related activity rather than of the isolated words or pictures frequently used in priming studies. Repeated activation of a densely interconnected network of concepts during a particular holiday season may have greater persistence than activation of an isolated concept. The effect was also found in academically relevant targets, which might be assumed to be salient throughout the school year, yet these items showed selective facilitation around finals. As was noted above, evidence for priming across contextual boundaries (e.g., Oliphant, 1983) has not always been observed in the literature, although in some cases low-frequency words do show such effects (e.g., Coane \& Balota, 2009; MacLeod, 1989). However, because the effects reported here were not frequency modulated, we suggest that the critical factor is whether the priming observed is for a single item or an item in an interconnected conceptual network. In fact, we believe that the present results should encourage researchers to extend their investigations beyond within-experiment manipulations to examine the influence of extraexperimental but tractable events on standard cognitive measures.

Given the rather large seasonal windows used, these effects might only approximate true seasonal influences. For example, Christmas targets were considered congruent about 3 weeks before Christmas because of the university break schedule. Furthermore, the types of materials used in this experiment (i.e., words) are not the most typical representation for holiday-related stimuli. Indeed, consider the Christmas holiday: Stores, public places, and homes are decorated with images of sleighs, reindeer, and trees. The verbal labels for these items are less common. Tasks such as lexical decision, which are more perceptual/ orthographic in nature, generally show reduced priming following modality changes, relative to more conceptual tasks, such as word association (e.g., Srinivas \& Roediger, 1990). Finally, it is likely that individual differences produce noise in these measures, such that the priming for Christmas-related items tended to be smaller in individuals who do not celebrate this holiday.

Although we have been discussing these findings primarily in terms of an activation mechanism, as was noted in the introduction, many priming effects can be explained by positing a retrieval mechanism rather than, or in addition to, an activation mechanism (e.g., Tenpenny, 1995). However, it is also noteworthy that given the different representational formats between the priming stimuli and the targets presented in the LDT and the relative sensitivity of retrieval processes to the similarity between encoding and retrieval events (Tulving \& Thomson, 1973), the phenomenon reported here does seem to reflect activation of conceptual-level information rather than priming of lexical forms. In this light, one might interpret the findings as more consistent with activation accounts of priming than with retrieval-based accounts. However, it should also be noted that a comparison of the effect sizes for the free recall task and the LDT does suggest that retrieval demands can exaggerate the seasonal priming effect, be- 
cause the effect in recall was substantially larger than that observed in the LDT. The fact that the congruency effect was observed in both the LDT and recall does raise the concern of contamination by conscious processes in the LDT. Although we cannot rule this out completely, it is important to consider that a minority of the trials were congruent, since only a few words related to each holiday were included. Thus, conscious retrieval would have been strongly discouraged in the present context, and the analysis of intrusions clearly indicated that response biases did not produce the observed effects. Ultimately, as in the case of standard repetition and semantic priming studies (see Bodner \& Masson, 1997; Bowers, 2000; Tenpenny, 1995), the present study cannot adjudicate between activationbased and episodic retrieval-based accounts. However, these results are the first to demonstrate that there are persistent changes in the accessibility of concepts (because of new episodic traces and/or activation of preexisting representations) as a function of time of the year.

\section{AUTHOR NOTE}

The experiment reported here was conducted in partial fulfillment of the requirements for the doctoral degree from Washington University in Saint Louis for J.H.C., who was supported by a Dean's Dissertation Fellowship. Funding was also provided by NIA Training Grant AG00030. We thank Jan Duchek and Mike Cortese for early input on these ideas, as well as Larry Jacoby, Mark McDaniel, and Roddy Roediger for support and feedback. Correspondence concerning this article should be addressed to J. H. Coane, Department of Psychology, Colby College, 5550 Mayflower Hill, Waterville, ME 04901 (e-mail: jhcoane@colby.edu).

\section{REFERENCES}

ANDERSON, J. R. (1983). A spreading activation theory of memory. Journal of Verbal Learning \& Verbal Behavior, 22, 261-295. doi:10.1016/ S0022-5371(83)90201-3

Balota, D. A., Cortese, M. J., Sergent-Marshall, S. D., Spieler, D. H., \& YAP, M. J. (2004). Visual word recognition of single-syllable words. Journal of Experimental Psychology: General, 133, 283-316. doi:10.1037/0096-3445.133.2.283

BaLota, D. A., \& LoRCH, R. F., JR. (1986). Depth of automatic spreading activation: Mediated priming effects in pronunciation but not in lexical decision. Journal of Experimental Psychology: Learning, Memory, \& Cognition, 12, 336-345. doi:10.1037/0278-7393.12.3.336

Balota, D. A., \& Spieler, D. H. (1999). Word frequency, repetition, and lexicality effects in word recognition tasks: Beyond measures of central tendency. Journal of Experimental Psychology: General, 128, 32-55. doi:10.1037/0096-3445.128.1.32

Balota, D. A., Yap, M. J., Cortese, M. J., Hutchison, K. A., KessLer, B., Loftis, B., ET AL. (2007). The English Lexicon Project. Behavior Research Methods, 39, 445-459.

BoDNER, G. E., \& MAsson, M. E. (1997). Masked repetition priming of words and nonwords: Evidence for a nonlexical basis for priming. Journal of Memory \& Language, 37, 268-293. doi:10.1006/jmla.1996.2507

BowERS, J. S. (2000). In defense of abstractionist theories of repetition priming and word identification. Psychonomic Bulletin \& Review, 7, 83-99.

CoAne, J. H., \& Balota, D. A. (2009). Repetition priming across event boundaries: Effects of lexical status, frequency, and testing conditions. Manuscript submitted for publication.

Collins, A. M., \& Loftus, E. F. (1975). A spreading-activation theory of semantic processing. Psychological Review, 82, 407-428. doi:10.1037/0033-295X.82.6.407

Coltheart, M., Davelaar, E., Jonasson, J. E., \& Besner, D. (1977). Access to the internal lexicon. In S. Dornic (Ed.), Attention and performance VI (pp. 535-555). Hillsdale, NJ: Erlbaum.
DeLL, G. S. (1986). A spreading-activation theory of retrieval in sentence production. Psychological Review, 93, 283-321. doi:10.1037/0033 $-295 X .93 .3 .283$

Faust, M. E., Balota, D. A., Spieler, D. H., \& Ferraro, F. R. (1999). Individual differences in information processing rate and amount: Implications for group differences in response latency. Psychological Bulletin, 125, 777-799. doi:10.1037/0033-2909.125.6.777

Green, M. W., \& Rogers, P. J. (1993). Selective attention to food and body shape words in dieters and restrained nondieters. International Journal of Eating Disorders, 14, 515-517. doi:10.1002/1098 -108X(199312)14:4<515::AID-EAT2260140417>3.0.CO;2-E

Hill, A. B., \& PAYnTer, S. (1992). Alcohol dependence and semantic priming of alcohol related words. Personality \& Individual Differences, 13, 745-750. doi:10.1016/0191-8869(92)90245-K

HinTZMAN, D. L. (1986). "Schema abstraction" in a multiple trace memory model. Psychological Review, 93, 411-428. doi:10.1037/0033 $-295 X .93 .4 .411$

MacLeod, C. M. (1989). Word context during initial exposure influences degree of priming in word fragment completion. Journal of Experimental Psychology: Learning, Memory, \& Cognition, 15, 398406. doi: $10.1037 / 0278-7393.15 .3 .398$

Meade, M. L., Watson, J. M., Balota, D. A., \& Roediger, H. L., III (2007). The roles of spreading activation and retrieval mode in producing false recognition in the DRM paradigm. Journal of Memory \& Language, 56, 305-320. doi:10.1016/j.jml.2006.07.007

Morris, C. D., Bransford, J. D., \& FranKs, J. J. (1977). Levels of processing versus transfer appropriate processing. Journal of Verbal Learning \& Verbal Behavior, 16, 519-533. doi:10.1016/S0022 $-5371(77) 80016-9$

NeELY, J. H. (1991). Semantic priming effects in visual word recognition: A selective review of current findings and theories. In D. Besner \& G. Humphreys (Eds.), Basic processes in reading: Visual word recognition (pp. 236-264). Hillsdale, NJ: Erlbaum.

Oliphant, G. W. (1983). Repetition and recency effects in word recognition. Australian Journal of Psychology, 35, 393-403. doi:10.1080 00049538308258751

Roediger, H. L., III, \& McDermott, K. B. (1995). Creating false memories: Remembering words not presented in lists. Journal of Experimental Psychology: Learning, Memory, \& Cognition, 21, 803-814. doi:10.1037/0278-7393.21.4.803

SRINIVAS, K., \& RoEdIGER, H. L., III (1990). Classifying implicit memory tests: Category association and anagram solution. Journal of Memory \& Language, 29, 389-412. doi:10.1016/0749-596X(90)90063-6

TenPenNy, P. L. (1995). Abstractionist versus episodic theories of repetition priming and word identification. Psychonomic Bulletin \& Review, 2, 339-363.

Tse, C.-S., \& NeELy, J. H. (2005). Assessing activation without source monitoring in the DRM paradigm. Journal of Memory \& Language, 53, 532-550. doi:10.1016/j.jml.2005.07.001

Tulving, E., \& Thomson, D. M. (1973). Encoding specificity and retrieval processes in episodic memory. Psychological Review, 80, 352373. doi:10.1037/h0020071

Whittlesea, B. W. A., \& JACOBY, L. L. (1990). Interaction of prime repetition with visual degradation: Is priming a retrieval phenomenon? Journal of Memory \& Language, 29, 546-565. doi:10.1016/0749 $-596 X(90) 90051-Z$

\section{NOTE}

1. When time of testing was included as a factor, a marginal interaction between congruency and time emerged in RTs $(p=.09)$ and in $z$-transformed RTs $(p=.06)$. The effect of congruency was present in the majority of the groups and was somewhat larger in some groups than in others. Because different numbers of participants were tested in each group, we interpret the interaction cautiously. The main point, of course, is that in almost all groups, congruent trials were faster than incongruent trials, suggesting replicability of the effect.

(Manuscript received January 24, 2009; revision accepted for publication June 19, 2009.) 